\title{
RACE RECOGNITION USING LOCAL DESCRIPTORS
}

\author{
Ghulam Muhammad, ${ }^{1, a}$, Muhammad Hussain ${ }^{2}$, Fatmah Alenezy $^{2}$, Anwar M. Mirzal, George Bebis ${ }^{2,3}$ \\ and Hatim Aboalsamh ${ }^{2}$ \\ ${ }^{1}$ Department of Computer Engineering, ${ }^{2}$ Department of Computer Science; College of Computer and \\ Information Sciences, King Saud University, Riyadh 11543, Saudi Arabia \\ ${ }^{3}$ Department of Computer Science and Engineering, University of Nevada at Reno \\ ${ }^{a}$ Email: ghulam@ksu.edu.sa
}

\begin{abstract}
This paper proposes a method for race recognition from face images using local descriptors. The proposed method uses two types of local descriptors: local binary pattern (LBP) and Weber local descriptors (WLD). First, LBP and WLD histograms are obtained separately from blocks of normalized face image. Kruskal-Wallis feature selection technique is applied to the histograms to select the significant bins for race recognition. Then the selected bins from the two histograms are concatenated block by block to produce the final feature set of the face image. Minimum city block distance is used as a classifier. The experiments are conducted using gray scale FERET images with five race groups. Experimental results show that the proposed method has superior race recognition accuracies for all the five race groups compared to LBP and WLD alone.
\end{abstract}

Index Terms - race recognition, local binary pattern, Weber local descriptors, face recognition

\section{INTRODUCTION}

There has been a growing interest to extract demographic information from face images due to several applications such as access control, surveillance, identity authentication, etc. Significant cognitive evidence supports that humans utilize information from various visual cues (e.g., gender, race, age, etc.) to identify a person. It is well known, for example, that people are more accurate at recognizing faces of their own race than faces of other races [1][2]. Therefore, categorizing faces into different race groups should help to reduce the search space as well as to increase the accuracy of person identification. In this paper, we concentrate on race recognition from faces. We often feel that people from other race look similar to each other than the people from our own race. MacLin and Malpass subjectively found that other race faces are encoded categorically and this categorization contributes to human perception [3]. Phillips et al analyze other-race effect on face recognition algorithms based on the result of Face Recognition Vendor Test (FRVT) 2006 [4]. They find that Western algorithms (developed by France, Germany and the United States research groups) recognized Caucasian faces more correctly than East Asian faces and East Asian algorithms (developed by China, Japan, and Korea research groups) recognized
East Asian faces more accurately than Caucasian faces. Two class (Asian and non-Asian) ethnicity (race) identification based on face images is proposed in [5]. The authors use multiscale LDA based classifier to classify 132 Asian faces and 131 non-Asian faces. They use a single Gaussian to model each face. An ensemble is constructed by integrating the classification results using dot product to find the final decision. An overall accuracy of $96.3 \%$ is achieved in their experiments. Hosoi et al [6] design ethnicity estimation method using Gabor wavelets transform and retinal sampling as features, and SVM as classifier. Three types of ethnic groups are classified: African, Asian, and European, and an overall approximately $94 \%$ accuracy is achieved. Zhiguang and Haizhou use LBP for demographic classification, which includes race, using face images [7]. AdaBoost algorithm is used on chi-square distant metric to form a strong classifier. Experimental results confirm that LBP features are comparable to Haar like features for Asian and non-Asian classification. However, the contribution of different types of LBP parameters is not studied in their work.

From the above discussion, it can be noted that race recognition using face images is not fully discovered with the state of the art features, though a significant progress has been made in face recognition. Some attempts are made for only two or three class problems, which is relatively easier than many class problems. Also no feature selection methods are applied to race recognition problems. Therefore, in this paper, we introduce a new race recognition method that (a) involves state of the art local features, (b) utilizes feature selection technique, and (c) works on five race groups (classes). The proposed method uses local binary pattern (LBP) [8] and Weber local descriptor (WLD) [9] as features, and city block distance measure as classifier.

The rest of the paper is organized as follows. Section 2 presents the proposed race recognition system, Section 3 shows experimental results with discussion, and finally Section 4 draws some conclusion.

\section{PROPOSED METHOD FOR RACE RECOGNITION}

Figure 1 shows a block diagram of the proposed race recognition system using face images. Two types of features, LBP and WLD, are extracted from the normalized 


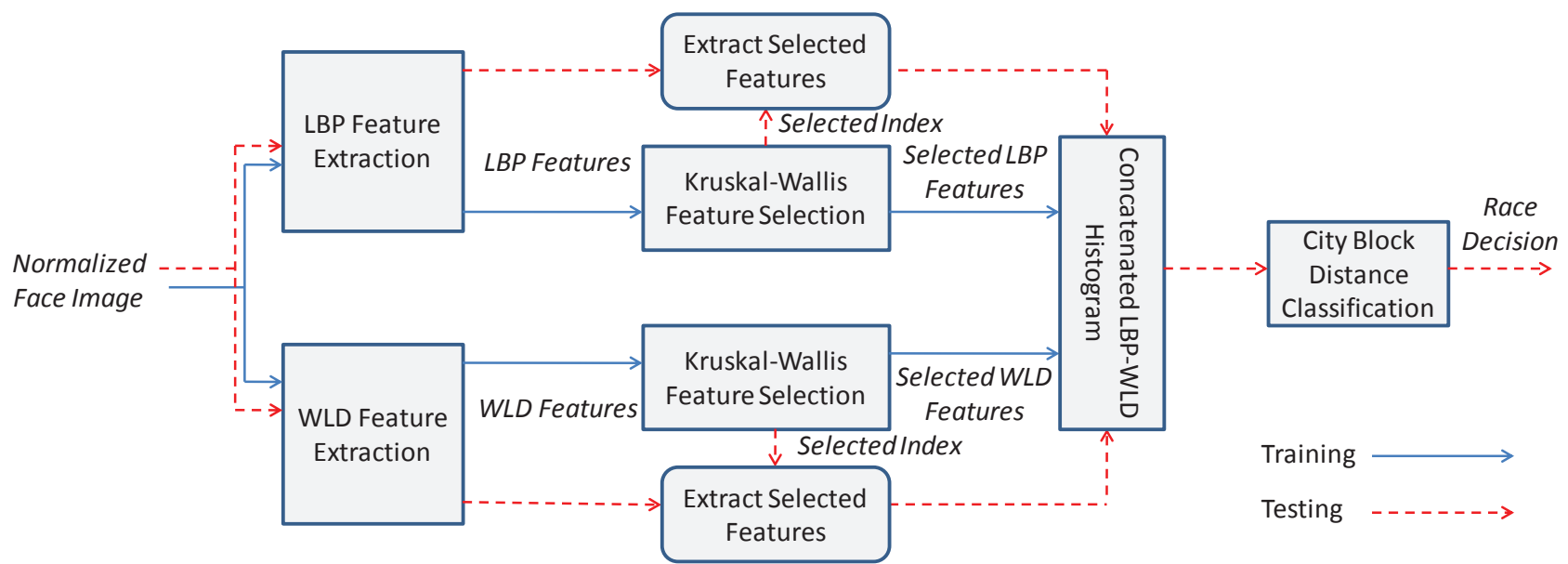

Figure 1. Block diagram of the proposed race recognition system.

input face images. Kruskal-Wallis feature selection method is then applied individually to each set of features to select highly discriminative features from LBP and WLD set. Optimized LBP and WLD histograms are concatenated to produce a combined feature set for the classifier. City block distance is calculated using training face images and test face image. The minimum distance is used to get the final decision.

\subsection{LBP}

LBP is one of the widely used and best performed texture descriptors in recent years [8]. This operator labels the pixels of an image by thresholding the $3 \times 3$-neighbourhood of each pixel with the center value and considering the result as a binary number. Then the histogram of the labels can be used as a texture descriptor. The operator can be extended to use neighborhood of different sizes. Specifically, LBP is computed by the following equation:

$$
L B P_{P, R}=\sum_{i=1}^{p-1} 2^{i} * S\left(p_{i}-p_{c}\right)
$$

where $p_{\mathrm{c}}$ is the center pixel and the thresholding operation is $S\left(p_{i}-p_{c}\right)=\left\{\begin{array}{ll}1 & p_{i}-p_{c} \geq 0 \\ 0 & p_{i}-p_{c}<0\end{array}\right.$. In the proposed race recognition method, the following parameters of LBP are varied: type of the LBP operator and block size. First parameter is $\mathrm{LBP}_{\mathrm{p}, \mathrm{R}}^{\text {mapping }}$, where mapping corresponds to using mapping table defined by one of the three mapping type options: 'u2' for uniform LBP, 'ri' for rotationinvariant LBP, and 'riu2' for uniform rotation-invariant LBP, while 0 is used for no mapping. LBP is called uniform if it contains at most two bitwise transitions from 0 to 1 or 1 to 0, e.g. 00000000, 11000011 and 00000111. The subscript represents $(\mathrm{P}, \mathrm{R})$ neighborhood, where $\mathrm{P}$ is the number of sampling points on circle with radius $\mathrm{R}$. The basic LBP method, which involves a $3 \times 3$ rectangular neighborhood, is also experimented. The second parameter is the block size, when the image is divided into number of blocks to localize the LBP histograms.

\subsection{WLD}

WLD is a recently developed robust and very powerful local descriptor [9]. It consists of two components: differential excitation and gradient orientation. It has been inspired by psychological law called Weber's Law. For calculation of WLD histogram, please refer to [9].

As mentioned in [9], this method has several advantages, such as: perfectly extracting the edges of image even if there is heavy noise, robust against changing in illumination and powerful representation ability. It is also reported that WLD outperforms LBP for highly texture images. However, WLD is not explored in face recognition applications yet. In WLD, there are three parameters that affect on optimizing the results: the number of dominant orientations $(\mathrm{T})$, the number of differential excitation segments $(\mathrm{M})$, and the number of bins in sub histogram segments $H_{\mathrm{m}, \mathrm{t}}(\mathrm{S})$. In the experiments in this paper, the values of the parameters are varied as the following $(T=6$ or $8 ; M=4$ or $6 ; S=10$ or $15)$.

\subsection{Kruskal-Wallis Feature Selection}

The number of bins in LBP and WLD histograms is very large, especially when they are calculated in blocks. Many of these bins may not contain sufficient discriminative information, and as a consequence may contribute to low performance of the recognition system. Also dealing with too many features slows down the classification process. In the proposed system, we introduce Kruskal-Wallis (KW) feature selection method, which is very simple to implement and involves less computation. KW method is a nonparametric one-way ANOVA (analysis of variance) test that can be applied to two or more classes. It tests the null hypothesis that the samples from two or more groups have equal medians, and returns $p$ value. If the $p$ value is close to zero for a certain feature, we select that feature for its 
discriminative power. On the other hand, if the $p$ value is far from zero for a certain feature, we discard the feature.

In the proposed method, during training, KW method is applied to LBP and WLD features separately. The features that have $p$ value less than a threshold are selected, and their indices are stored for testing. During testing, the features with those indices are selected for classification.

\subsection{LBP and WLD Histograms Concatenation}

LBP and WLD extract different types of information from the face images. This is evident from the misrecognition face pairs using LBP and WLD separately. In the experiments, it is found that only $15 \%$ of the misrecognition face pairs are common in both LBP and WLD based race recognition. This finding suggests that if LBP and WLD are fused, their complementary information can lead to a better race recognition performance.

After optimizing the LBP and the WLD histograms by using $\mathrm{KW}$ technique, they are concatenated to produce a combined histogram. Figure 2 demonstrates such a histogram concatenation. Figure 2(a) represents an optimized LBP histogram for a certain face image, 2(b) corresponds to an optimized WLD histogram for that image, while 2(c) shows the concatenated histogram. From the LBP and WLD histograms it is clear that they produce different types of information for the same image.

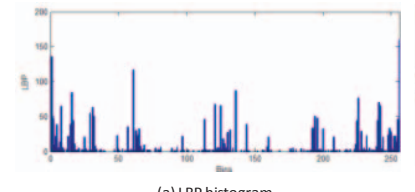

(a) LBP histogram

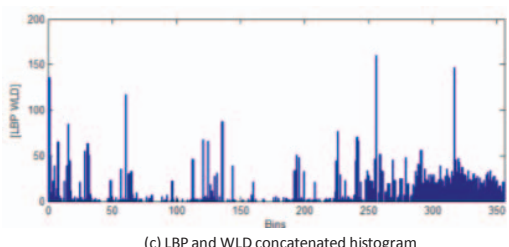

Figure 2. LBP and WLD histogram concatenation.

\section{EXPERIMENTS}

In the experiments, FERET database [10] is used. There are eight race groups in the database. The race groups are: White, Asian-Middle-Eastern (Middle), Asian, Hispanic, Black-or-African-American (Black), Pacific-Islander, Native-American, and other. The number of subjects in each group of Pacific-Islander, Native-American, and other is less than 10. Therefore, we study the other five major race groups, each of which contains more than 50 subjects. In our experiments, we focus on two sets of gray image database: $f a$ and $f b$. $f a$ set includes 1,204 frontal images with regular expression. This set of images are called gallery images and used for training. $f b$ set includes 1,195 images of the same subjects in $f a$ set but with alternative facial expression. The major five race group consists of 1180 images. The $\mathrm{fb}$ set is termed as probe set and used for testing. All the face images are normalized and cropped to $60 \times 48$ pixel sizes. City block minimum distance classifier (L1) is used in the experiments.

First, race recognition performances of principal component analysis (PCA), LBP, and WLD are evaluated using the whole image size. Figure 3 shows the result. Bracket values in the legend shows the parameters for the best results of the corresponding methods. Figure shows that PCA with 200 principal components performs better than LBP and WLD when the whole image size is used. It is understandable that local descriptors like LBP and WLD cannot exploit details of local regions when they are applied to the whole image size at a time. Second, LBP and WLD performances using different block sizes are evaluated. Table 1 shows the results. Considering all the five race groups, block size $10 \times 16$ produces the best results for both LBP and WLD. LBP performs better than WLD for Asian, Black, Hispanic, and Middle groups, while the reverse is true for White group. The number of features of these LBP and WLD is 4608 and 2880, respectively.

The results of KW feature selection technique on these best LBP and WLD features are shown in Table 2. The optimum performance is obtained with $\mathrm{p}=0.16$ for LBP and $\mathrm{p}=0.15$ for WLD. The feature selection techniques significantly reduces the size of the feature vector of LBP from 4608 to 1633 and of WLD from 2880 to 1632 without decreasing the accuracies much.

The proposed method that concatenates optimum bins from LBP and WLD produces the best results in all the five race categories as shown in Table 3 . The best results are Asian: 99.64\%, Black: 98.78\%, Hispanic: 96.57\%, Middle: $100 \%$, and White: $100 \%$. These results suggest that LBP and WLD contain complementary information for race recognition, and their fusion provides the best performance.

A time comparison between LBP, WLD, and the proposed method is shown in Table 4 . The time shown in Table 4 is per face image and in seconds. Training time includes time to generate the histogram and to select the bins (in case of KW method), while testing time includes time to generate the histogram and to find distances between the test image histogram and all other images histograms to obtain the minimum distance index.

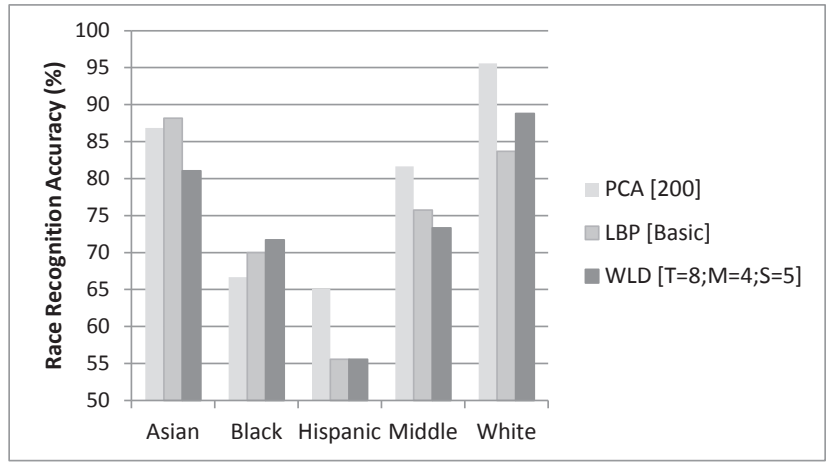

Figure 3. Race recognition accuracies (\%) of different methods using the whole image size. 
Table 1. Race recognition accuracies (\%) of different methods using different block sizes. The best parameters of LBP and WLD (T,M,S) are also mentioned.

\begin{tabular}{|c|c|c|c|c|c|c|}
\hline $\begin{array}{c}\text { Block } \\
\text { size }\end{array}$ & $\begin{array}{l}\text { Me- } \\
\text { thod }\end{array}$ & Asian & Black & $\begin{array}{l}\text { Hisp- } \\
\text { anic }\end{array}$ & Middle & White \\
\hline \multirow{2}{*}{$20 \times 16$} & $\begin{array}{c}\text { LBP } \\
\text { (Basic) }\end{array}$ & 97.37 & 92.93 & 93.65 & 98.33 & 97.66 \\
\hline & $\begin{array}{l}\text { WLD } \\
(8,6,5)\end{array}$ & 94.74 & 91.92 & 92.06 & 90.00 & 98.18 \\
\hline \multirow[b]{2}{*}{$20 \times 12$} & $\mathrm{LBP}_{8,1}{ }^{0}$ & 95.26 & 95.96 & 92.06 & 95.00 & 97.79 \\
\hline & $\begin{array}{l}\text { WLD } \\
(8,4,5)\end{array}$ & 94.74 & 95.96 & 92.06 & 93.33 & 99.35 \\
\hline \multirow{2}{*}{$10 \times 16$} & $\begin{array}{c}\text { LBP } \\
\text { (Basic) }\end{array}$ & 97.89 & 97.98 & 93.65 & 100 & 98.18 \\
\hline & $\begin{array}{c}\text { WLD } \\
(8,4,5)\end{array}$ & 96.32 & 96.97 & 92.06 & 98.33 & 99.74 \\
\hline \multirow{2}{*}{$10 \times 12$} & $\begin{array}{c}\text { LBP } \\
\text { (Basic) }\end{array}$ & 97.37 & 97.98 & 92.06 & 98.33 & 98.44 \\
\hline & $\begin{array}{l}\text { WLD } \\
(6,4,5)\end{array}$ & 96.84 & 96.97 & 88.89 & 96.67 & 99.22 \\
\hline
\end{tabular}

Table 2. Race recognition accuracies (\%) of LBP and WLD after applying Kruskal-Wallis feature selection technique.

\begin{tabular}{|c|c|c|c|c|c|c|}
\hline $\begin{array}{c}p- \\
\text { value }\end{array}$ & $\begin{array}{c}\text { No. of } \\
\text { features }\end{array}$ & Asian & Black & $\begin{array}{c}\text { Hispa- } \\
\text { nic }\end{array}$ & Middle & White \\
\hline $\begin{array}{c}0.16 \\
\text { (LBP) }\end{array}$ & 1633 & 98.42 & 95.96 & 93.65 & 100.00 & 98.18 \\
\hline $\begin{array}{c}0.15 \\
\text { (WLD) }\end{array}$ & 1632 & 97.74 & 96.89 & 92.06 & 98.33 & 99.53 \\
\hline
\end{tabular}

Table 3. Comparative race recognition accuracies (\%) of different methods including the proposed method.

\begin{tabular}{|l|l|l|l|l|l|l|}
\hline \multirow{2}{*}{ Methods } & No. of & \multicolumn{5}{|c|}{ Accuracy (\%) } \\
\cline { 3 - 7 } & features & Asian & Black & $\begin{array}{c}\text { Hispa- } \\
\text { nic }\end{array}$ & Middle & White \\
\hline LBP & 4608 & 97.89 & 97.98 & 93.65 & 100 & 98.18 \\
\hline LBP+KW & 1633 & 98.42 & 95.96 & 93.65 & 100 & 98.18 \\
\hline WLD & 2880 & 96.32 & 96.97 & 92.06 & 98.33 & 99.74 \\
\hline $\begin{array}{l}\text { WLD+K } \\
\text { W }\end{array}$ & 1632 & 97.74 & 96.89 & 92.06 & 98.33 & 99.53 \\
\hline $\begin{array}{l}\text { Proposed } \\
\text { Method }\end{array}$ & 3265 & 99.64 & 98.78 & 96.57 & 100 & 100 \\
\hline
\end{tabular}

Table 4. Elapsed time in seconds for different methods.

\begin{tabular}{|c|c|c|}
\hline Methods & $\begin{array}{c}\text { Training } \\
\text { (histogram } \\
\text { generation and / } \\
\text { or selection) }\end{array}$ & $\begin{array}{c}\text { Testing } \\
\text { (histogram } \\
\text { generation and } \\
\text { classification) }\end{array}$ \\
\hline LBP & 0.1363 & 0.1394 \\
\hline LBP + KW & 0.1597 & 0.1378 \\
\hline WLD & 0.1952 & 0.1979 \\
\hline WLD + KW & 0.2161 & 0.1968 \\
\hline Proposed Method & 0.7073 & 0.3337 \\
\hline
\end{tabular}

\section{CONCLUSION}

LBP and WLD based race recognition using face images is proposed. In the experiments the following conclusions are obtained:

- The proposed method outperforms PCA, LBP, and WLD in all race groups in terms of accuracy.

- LBP gives better results than WLD in all race groups except White. However, the difference between their recognition performances is less.

- By applying KW feature selection, comparable accuracies can be obtained with one-third features in case of LBP and half number of features in case of WLD.

- Concatenating optimized LBP and WLD bins gives higher recognition rates than LBP and WLD alone.

The proposed method will be extended in a future study by applying other powerful feature selection methods and by assigning different weights in different blocks.

\section{Acknowledgement}

This work is supported by the National Plan for Science and Technology, King Saud University, Riyadh, Saudi Arabia under project number 10-INF1044-02.

\section{REFERENCES}

[1] Brigham, J., "The influence of race on face recognition", Aspects of face processing (H. Ellis, M. Jeeves, and F. Newcombe, eds), pp. 170-177, 1986.

[2] O'Toole, A. et al., "Another-race effect for classifying faces by sex", Perception, vol. 25, pp. 669-676, 1996.

[3] MacLin, O.H. and Malpass, R.S., "Racial categorization of faces: The ambiguous race face effect", Psychology, Public Policy, and Law, vol. 7, no. 1, pp. 98-118, 2001.

[4] Phillips, P. J., et al., "An Other-Race Effect for Face Recognition Algorithms", ACM Transactions on Applied Perception (TAP), vol. 8, Issue 2, January 2011.

[5] Lu, X. and Jain, A.K., "Ethnicity Identification from Face Images", Proc. of SPIE International Symposium on Defense and Security, pp. 114-123, 2004.

[6] Hosoi, S., et al., "Ethnicity Estimation with Facial Images," Proc. 6th IEEE Int. Conf, on Automatic Face and Gesture Recognition (AFGR), pp. 195-200, 2004.

[7] Zhiguang Y., and Haizhou A., "Demographic Classification with Local Binary Patterns", Proc. International Conf. on Biometrics (ICB), pp.464-473, 2007.

[8] Ahonen, T., et al., "Face description with local binary patterns: Application to face recognition," IEEE Trans. Pattern Analysis and Machine Intelligence, vol. 28, pp. 2037-2041, 2006.

[9] Chen, J., et al., "WLD: A Robust Local Image Descriptor," IEEE Trans. Pattern Analysis and Machine Intelligence, , vol. 32, no. 9, pp. 1705-1720, Sep. 2010.

[10] Phillips, P.J., et al., "The FERET evaluation methodology for face-recognition algorithms," IEEE Trans. Pattern Analysis and Machine Intelligence, , vol. 22, no. 10, pp. 1090- 1104, Oct. 2000. 